\title{
Final treatment outcomes of delamanid- containing regimens in patients with MDR-/XDR-TB in South Korea
}

\author{
To the Editor:
}

A phase III trial of delamanid showed acceptable safety and tolerability; however, it failed to demonstrate superior efficacy of delamanid in terms of reduction in time to sputum conversion and improvement in final treatment outcomes compared with placebo [1]. How to interpret these results and identify the role of delamanid in multidrug-resistant tuberculosis (MDR-TB) treatment is challenging [2]. Despite the disappointing results, this trial suggests that more studies are needed to better understand the role of delamanid in the current management of MDR-TB. So far, there have been several reports of real-world data on the interim outcomes of delamanid treatment [3-6], but few on its final outcomes [7].

We previously reported that delamanid-containing regimens achieved 95\% culture conversion at 6 months with acceptable safety and tolerability [5]. Following these encouraging interim results, we investigated the final treatment outcomes of the MDR-TB cohort treated with delamanid-containing regimens.

In South Korea, a total of 33796 patients with TB, 618 with MDR-TB and 37 with extensively drug-resistant tuberculosis (XDR-TB) were notified in 2018 [8]. Delamanid has been marketed since November 2015 in South Korea. National medical insurance provides reimbursement for the total cost of 24 weeks of delamanid treatment. However, off-label use of delamanid, including use for more than 24 weeks, is strictly prohibited and concurrent use of delamanid and bedaquiline was also discouraged during the study periods. We retrospectively evaluated patients who received delamanid for at least 1 month from 1 November 2015 to 31 December 2017, at six university affiliated hospitals and one national TB hospital in South Korea. To evaluate the final treatment outcomes, we included patients who received delamanid within 6 months of initiation of MDR-TB treatment and had final treatment outcomes as of 31 January 2019.

Patient selection and design of the treatment regimen was independently carried out at each hospital in accordance with World Health Organization (WHO) guidelines [9]. The anti-TB agents were administered under directly observed therapy during hospitalisation and subsequently self-administered after discharge. Delamanid was administered at a dose of $100 \mathrm{mg}$ twice daily for 24 weeks. Drug susceptibility testing was conducted for 15 anti-TB drugs using an absolute concentration method in the Korean Institute of Tuberculosis, Osong, South Korea. Treatment outcomes were assessed according to WHO definitions; "treatment success" was defined as the sum of "cured" and "treatment completed"; all other outcomes were defined as "unfavourable outcomes" [10].

Of 72 patients who received delamanid during the study period, nine were excluded as treatment was still on-going as of 31 January 2019 and 14 were excluded as delamanid was started after 6 months from initiation of MDR-TB treatment. Thus, a total of 49 patients were included, of whom 27 had been included in the earlier interim analysis [5]. The median age of the patients was 47 years (interquartile range (IQR) 32-58 years), 29 were male (59.2\%), and all were HIV negative (table 1). All patients had pulmonary TB, of whom three (6.1\%) had concomitant extrapulmonary TB. The proportion of pre-XDRor XDR-TB patients was $71.4 \%$ (35 out of 49 ). When delamanid was initiated, 16 patients (32.7\%) were

@ERSpublications

The optimised use of delamanid combined with effective repurposed drugs could achieve a high treatment success rate, even in pre-extensively drug-resistant (XDR)-TB and XDR-TB patients http://bit.ly/2plvEOT

Cite this article as: Mok J, Kang H, Koh W-J, et al. Final treatment outcomes of delamanid-containing regimens in patients with MDR-/XDR-TB in South Korea. Eur Respir J 2019; 54: 1900811 [https://doi.org/ 10.1183/13993003.00811-2019]. 
TABLE 1 Characteristics and treatment outcomes of 49 multidrug-resistant tuberculosis (MDR-TB) patients according to baseline drug resistance pattern

\begin{tabular}{|c|c|c|c|c|c|c|}
\hline Characteristics & Total & MDR-TB ${ }^{\#, n}$ & $\begin{array}{l}\text { SLID-resistant } \\
\text { pre-XDR-TB }\end{array}$ & $\begin{array}{l}\text { FQ-resistant } \\
\text { pre-XDR-TB }\end{array}$ & XDR-TB & $\mathrm{p}$-value \\
\hline Subjects $n$ & 49 & 14 & 7 & 20 & 8 & \\
\hline Age years & $47.0(32.0-58.0)$ & $40.5(30.0-58.3)$ & $51.0(41.0-64.0)$ & $52.5(38.5-58.0)$ & $37.0(24.8-57.0)$ & 0.433 \\
\hline Body mass index $\mathrm{kg} \cdot \mathrm{m}^{-2}$ & $20.7(18.6-23.2)$ & $21.7(19.6-24.0)$ & $19.1(17.1-25.0)$ & $19.8(16.7-21.8)$ & $21.9(18.6-23.3)$ & 0.270 \\
\hline Previous TB treatment & & & & & & 0.147 \\
\hline Second-line drug & $20(40.8)$ & $3(21.4)$ & $3(42.9)$ & $12(60.0)$ & $2(25.0)$ & \\
\hline Sputum AFB smear positive ${ }^{\S}$ & $16(32.7)$ & $1(7.1)$ & $3(42.9)$ & $8(40.0)$ & $4(50.0)$ & 0.081 \\
\hline Sputum MTB culture positive ${ }^{\S}$ & 29 (59.2) & $2(14.3)$ & $4(57.1)$ & $17(85.0)$ & $6(75.0)$ & $<0.001$ \\
\hline \multicolumn{7}{|l|}{ Radiological finding } \\
\hline Bilateral lung involvement & $29(59.2)$ & $6(42.9)$ & $3(42.9)$ & 15 (75.0) & $5(62.5)$ & 0.216 \\
\hline Cavity & $27(55.1)$ & $6(42.9)$ & $1(14.2)$ & 14 (70.0) & $6(75.0)$ & 0.040 \\
\hline Linezolid & 31 (63.3) & $3(21.4)$ & $5(71.4)$ & $15(75.0)$ & $8(100.0)$ & 0.001 \\
\hline Clofazimine & $17(34.7)$ & $4(28.6)$ & $1(14.2)$ & $6(30.0)$ & $6(75.0)$ & 0.076 \\
\hline Meropenem/clavulanic acid & $6(12.2)$ & $1(7.1)$ & $0(0.0)$ & $4(20.0)$ & $1(12.5)$ & 0.716 \\
\hline Number of companion drugs ${ }^{f}$ & $5.0(4.0-6.0)$ & $5.0(4.0-5.3)$ & $4.0(4.0-5.0)$ & $5.0(4.3-6.0)$ & $5.0(5.0-6.8)$ & 0.151 \\
\hline $\begin{array}{l}\text { Number of drugs after delamanid } \\
\text { discontinuation }\end{array}$ & $5.0(4.0-5.0)$ & $4.0(3.0-5.0)$ & $5.0(4.0-6.0)$ & $5.0(4.0-5.0)$ & $5.0(4.0-5.8)$ & 0.058 \\
\hline Total treatment duration days & $\begin{array}{c}607.0 \\
(538.5-719.5)\end{array}$ & $\begin{array}{c}566.0 \\
(436.0-667.3)\end{array}$ & $\begin{array}{c}630.0 \\
(564.0-729.0)\end{array}$ & $\begin{array}{c}607.0 \\
(539.5-712.5)\end{array}$ & $\begin{array}{c}724.5 \\
(555.3-734.0)\end{array}$ & 0.402 \\
\hline $\begin{array}{l}\text { Surgical resection } \\
\text { Treatment outcome }\end{array}$ & $2(4.1)$ & $1(7.1)$ & $0(0.0)$ & $1(5.0)$ & $0(0.0)$ & $>0.999$ \\
\hline Treatment success & $40(81.6)$ & 12 (85.7) & $6(85.7)$ & 15 (75.0) & $7(87.5)$ & 0.870 \\
\hline Cured & $39(79.6)$ & $11(78.6)$ & $6(85.7)$ & $15(75.0)$ & 7 (87.5) & \\
\hline Treatment completed & $1(2.0)$ & $1(7.1)$ & $0(0.0)$ & $0(0.0)$ & $0(0.0)$ & \\
\hline
\end{tabular}

Data are presented as $\mathrm{n}(\%)$ or median (interquartile range), unless otherwise stated. SLID: second-line injectable drug; XDR: extensively drug-resistance FQ: fluoroquinolone; AFB: acid fast bacilli; MTB: Mycobacterium tuberculosis. " : including one patient with rifampicin-resistant TB; ${ }^{\uparrow}$ : MDR-TB without additional resistance to fluoroquinolones and second-line injectable drugs; ${ }^{+}$: comparison between four groups; ${ }^{\S}$ : at delamanid initiation; ${ }^{f}$ : drug included in the initial treatment regimen with delamanid.

smear positive and 29 (59.2\%) were culture positive. Delamanid was started at a median of 6 days (IQR 627 days) from the initiation of the MDR-TB regimen.

At 24 weeks of delamanid treatment, the proportion of culture conversion was 96.6\% (28 out of 29); $95.8 \%$ ( 23 of 24 ) in liquid medium and $96.3 \%$ (26 of 27) in solid medium. All 20 patients with negative cultures at delamanid initiation maintained a culture-negative status at 24 weeks of delamanid treatment. The median time to culture conversion using liquid medium was 44.5 days (IQR 19.5-78.3 days) and that using solid medium was 42.0 days (IQR 17.0-60.0 days). In 16 patients with smear-positive sputum at delamanid initiation, the median time to smear conversion was 43.5 days (IQR 19.8-80.5 days).

Of the 49 patients, $40(81.6 \%)$ achieved treatment success, and the treatment success rate was not significantly different among the groups according to baseline drug resistance pattern. Patients were treated with a median of five companion drugs for a total median of 20 months. Number of drugs used and total treatment duration were not significantly different among the groups. The most common companion drug was cycloserine $(73.5 \%)$, followed by linezolid $(63.3 \%)$. Linezolid was used more frequently in the fluoroquinolone (FQ)-resistant pre-XDR- and XDR-TB group compared with the MDR-TB group. 30 patients were followed up after the end of treatment for a median of 201 days (IQR 103.5-311.5 days) and none of them showed recurrence. 
Nine patients (18.4\%) had unfavourable outcomes. Three patients died during treatment. Causes of death were respectively pneumonia, possible sudden cardiac death, and acute myocardial infarction. They occurred at 1, 1, and 3 months after completion of delamanid, respectively. These patients were culture-negative at the time of death and delamanid was considered unlikely to be related to all deaths. Treatment failure occurred in three patients; one patient did not convert by the end of the intensive phase and two patients reverted back to culture positive in the continuation phase. Three patients with other outcomes (one lost to follow-up and two not evaluated) maintained culture-negative status at the time of each outcome.

Three patients experienced QTcF interval of $>500 \mathrm{msec}$ at 4 weeks $(n=1)$ and 12 weeks $(n=2)$ from delamanid initiation. One patient continued delamanid, while two patients transiently discontinued delamanid. These patients were monitored more closely with an at least weekly ECG. After returning to a QTcF interval of $<480 \mathrm{msec}$, delamanid was reintroduced without significant adverse events or further QT prolongation. No clinically significant arrhythmia or event was observed in any of the included patients. Except QT prolongation, there were no serious adverse events or adverse drug reactions above grade 3 related to delamanid. All 49 patients completed the 24 weeks of delamanid treatment.

This study showed a delamanid-containing regimen could achieve a high treatment success rate in difficult-to-treat MDR-/XDR-TB patients. Interestingly, treatment success rate was not significantly different among patients with MDR-, pre-XDR- and XDR-TB. MDR-TB patients have quite different treatment outcomes, as per the resistance to core drugs, i.e. MDR-, pre-XDR- and XDR-TB $[11,12]$. Recently, treatment success rates of FQ-susceptible MDR-TB treated with conventional regimens without new drugs, such as delamanid and bedaquiline, have improved in several settings: $83.2 \%$ in a Korean multicentre study [13] and $79.8 \%$ in the STREAM trial [14]. The treatment success rate of $81.6 \%$ in our study and $84.2 \%$ in Latvia [7] were comparable with success rates in FQ-susceptible MDR-TB, despite the larger proportion of FQ-resistant pre-XDR-TB and XDR-TB. However, these comparisons should be carefully interpreted because of the difference in study populations; our study population is an HIV-uninfected population. In our study, there were no significant differences in treatment success rate, number of drugs used and total treatment duration among the groups according to baseline drug resistance pattern. This finding suggests that optimised use of delamanid could help to overcome the gap in treatment success rate between MDR-TB and XDR-TB, with the same number of companion drugs and similar treatment durations. This study could not demonstrate the efficacy of delamanid alone in MDR-TB treatment. We believe that optimised use of delamanid depends on appropriate patient selection as well as effective companion drugs. Repurposed drugs such as linezolid and clofazimine could be effective companion drugs to maintain the efficacy of a delamanid-containing regimen. In our study, linezolid was used in $63.3 \%$ of the 49 patients and more frequently in the FQ-resistant pre-XDR- and XDR-TB group than in the MDR-TB group.

Our study has limitations due to its retrospective nature and relatively small sample size. However, our results suggest that the optimised use of delamanid combined with effective repurposed drugs could achieve a high treatment success rate, even in pre-XDR-TB and XDR-TB patients.

Jeongha Mok ${ }^{1}$, Hyungseok Kang ${ }^{2}$, Won-Jung Koh $\oplus^{3}$, Byung Woo Jhun $\oplus^{3}$, Jae-Joon Yim ${ }^{4}$, Nakwon Kwak ${ }^{4}$, Taehoon Lee $\oplus^{5}$, Bohyoung Kang ${ }^{6}$ and Doosoo Jeon ${ }^{7}$

${ }^{1}$ Dept of Internal Medicine, Pusan National University Hospital, Pusan National University College of Medicine, Busan, South Korea. ${ }^{2}$ Dept of Chest Medicine, Masan National Tuberculosis Hospital, Masan, South Korea. ${ }^{3}$ Division of Pulmonary and Critical Care Medicine, Dept of Medicine, Samsung Medical Center, Sungkyunkwan University School of Medicine, Seoul, South Korea. ${ }^{4}$ Division of Pulmonary and Critical Care Medicine, Dept of Internal Medicine, Seoul National University College of Medicine, Seoul, South Korea. ${ }^{5}$ Dept of Internal Medicine, Ulsan University Hospital, University of Ulsan College of Medicine, Ulsan, South Korea. ${ }^{6}$ Dept of Internal Medicine, Dong-A University Hospital, Busan, South Korea. ${ }^{7}$ Dept of Internal Medicine, Pusan National University Yangsan Hospital, Pusan National University College of Medicine, Yangsan, South Korea.

Correspondence: Doosoo Jeon, Dept of Internal Medicine, Pusan National University Yangsan Hospital, 20 Geumo-ro, Yangsan 50612, South Korea. E-mail: sooli10@hanmail.net

Received: 23 April 2019 | Accepted after revision: 30 June 2019

Conflict of interest: None declared.

\section{References}

1 von Groote-Bidlingmaier F, Patientia R, Sanchez E, et al. Efficacy and safety of delamanid in combination with an optimised background regimen for treatment of multidrug-resistant tuberculosis: a multicentre, randomised, double-blind, placebo-controlled, parallel group phase 3 trial. Lancet Respir Med 2019; 7: 249-259.

2 Pontali E, Centis R, D'Ambrosio L, et al. Recent evidence on delamanid use for rifampicin-resistant tuberculosis. J Thorac Dis 2019; 11: Suppl. 3, S457-S460. 
3 Hafkin J, Hittel N, Martin A, et al. Early outcomes in MDR-TB and XDR-TB patients treated with delamanid under compassionate use. Eur Respir J 2017; 50: 1700311.

4 Hewison C, Ferlazzo G, Avaliani Z, et al. Six-month response to delamanid treatment in MDR TB patients. Emerg Infect Dis 2017; 23: 1746-1748.

5 Mok J, Kang H, Hwang SH, et al. Interim outcomes of delamanid for the treatment of MDR- and XDR-TB in South Korea. J Antimicrob Chemother 2018; 73: 503-508.

6 Mohr E, Hughes J, Reuter A, et al. Delamanid for rifampicin-resistant tuberculosis: a retrospective study from South Africa. Eur Respir J 2018; 51: 1800017.

7 Kuksa L, Barkane L, Hittel N, et al. Final treatment outcomes of multidrug- and extensively drug-resistant tuberculosis patients in Latvia receiving delamanid-containing regimens. Eur Respir J 2017; 50: 1701105.

8 Korea Centers for Disease Control and Prevention. Annual Report on the Notified Tuberculosis Patients in Korea 2018. Cheongwon, Korea Centers for Disease Control and Prevention, 2019. http://tbzero.cdc.go.kr/tbzero/board/ boardList.do Date last updated: 2019. Date last accessed: April 15, 2019.

9 World Health Organization. Companion handbook to the WHO guidelines for the programmatic management of drug-resistant tuberculosis. WHO/HTM/TB/2014.11. Geneva, WHO Press, 2014. www.who.int/tb/publications/ pmdt_companionhandbook/en/ Date last updated: 2014. Date last accessed: April 15, 2019.

10 World Health Organization. Definitions and reporting framework for tuberculosis-2013 revision. WHO/HTM/ TB/2013.2. Geneva, WHO Press, 2013. http://apps.who.int/iris/handle/10665/79199 Date last updated: 2013. Date last accessed: April 15, 2019.

11 Falzon D, Gandhi N, Migliori GB, et al. Resistance to fluoroquinolones and second-line injectable drugs: impact on multidrug-resistant TB outcomes. Eur Respir J 2013; 42: 156-168.

12 Ahmad N, Ahuja SD, Akkerman OW, et al. Treatment correlates of successful outcomes in pulmonary multidrug-resistant tuberculosis: an individual patient data meta-analysis. Lancet 2018; 392: 821-834.

13 Kang YA, Shim TS, Koh WJ, et al. Choice between levofloxacin and moxifloxacin and multidrug-resistant tuberculosis treatment outcomes. Ann Am Thorac Soc 2016; 13: 364-370.

14 Nunn AJ, Phillips PPJ, Meredith SK, et al. A trial of a shorter regimen for rifampin-resistant tuberculosis. $N$ Engl J Med 2019; 380: 1201-1213. 